\title{
Straightforward access to bisbenzamidine DNA binders and their use as versatile adaptors for DNA-promoted processes
}

Mateo I. Sánchez, Olalla Vázquez, José Martínez-Costas, M. Eugenio Vázquez and José L. Mascareñas

\section{Accepted Manuscript}

\section{How to cite:}

Sánchez, M., Vázquez, O., Martínez-Costas, J., Vázquez, M., \& Mascareñas, J. (2012). Straightforward access to bisbenzamidine DNA binders and their use as versatile adaptors for DNApromoted processes. Chem. Sci. 3, 2383-2387. doi: 10.1039/c2sc00027j

\section{Copyright information:}

(C) The Royal Society of Chemistry 2012 


\title{
Straightforward access to fluorogenic bisbenzamidine DNA binders and their use as versatile adaptors for DNA-promoted processes
}

\author{
Mateo I. Sánchez, ${ }^{\dagger}$ Olalla Vázquez, ${ }^{\dagger}$ José Martínez-Costas, ${ }^{\circledR}$ M. Eugenio Vázquez ${ }^{\dagger} *$ and \\ José L. Mascareñas ${ }^{\dagger} *$ \\ ${ }_{5}$ Received (in $\left.X X X, X X X\right)$ th $X X X X X X X X X 20 X X$, Accepted $X$ th $X X X X X X X X X 20 X X$ \\ DOI: 10.1039/b000000x
}

Bis-benzamidines are an important family of minor groove DNA-binding agents. We present a one-step synthesis of aromatic aza-bisbenzamidines that allows the straightforward and versatile access to a large variety of these molecules. One of them, the fluorogenic azide-aza-bisbenzamidine 13, can be readily 10 modified via click-chemistry with a variety of functionalities that can therefore be delivered to the vicinity of an A/T-rich DNA minor groove. This strategy therefore provides a simple means for triggering site selective DNA-promoted biochemical and physicochemical processes.

\section{Introduction}

A major research goal at the frontier between chemistry and 15 biomedicine is the efficient and specific targeting of double stranded DNA with small molecules. Towards this end, a wide range of systems capable of binding to the DNA minor groove has been described over the years. ${ }^{1}$ In addition to well-known $\beta$ hairpin polyamides that display very good DNA affinities and 20 sequence specificities, ${ }^{2}$ bisbenzamidines, such as propamidine (1), pentamidine (3) of furamidine (5), represent simple but relevant alternatives for binding to A/T-rich sites, ${ }^{3}$ and have found important applications as antiparasitic agents (Figure 1). ${ }^{4}$



Fig 1 Structures of propamidine (1), pentamidine (3), their aza versions 2 and 4 , and furamidine (5).

Following our discovery that the aza-propamidine derivative 2 displays strong fluorogenicity when binding to short A/T-rich 30 sequences, ${ }^{5}$ we considered that a simple and versatile access to this type of molecules might provide new opportunities for discovering chemical, biophysical or biochemical processes triggered by specific DNA recognition events. ${ }^{6}$ In particular, we were called by the possibility of equipping these DNA binders 35 with orthogonal handles to attach selected chemical cargoes, which could thus be delivered to the vicinity of specific DNA sites. It is surprising that, despite the extensive study of DNA binding processes, there are still very few precedents on the functional modification of the non-interacting face of synthetic ${ }_{40}$ DNA binders. ${ }^{7}$ Herein we describe a straightforward entry to a series of aza-bisbenzamidines featuring aromatic rings between the benzamidine units, including the phenylazido-derivative 13. This compound must display the azido group protruding out of the DNA surface when bound to the DNA minor groove, and 45 therefore represents a valuable platform for delivering designed components to specific DNA locations. ${ }^{8}$ We describe the use of this derivative for the synthesis of three conjugates in which the appendages provide for obtaining different $\mathrm{A} / \mathrm{T}$ specific, dsDNApromoted responses (Figure 2).

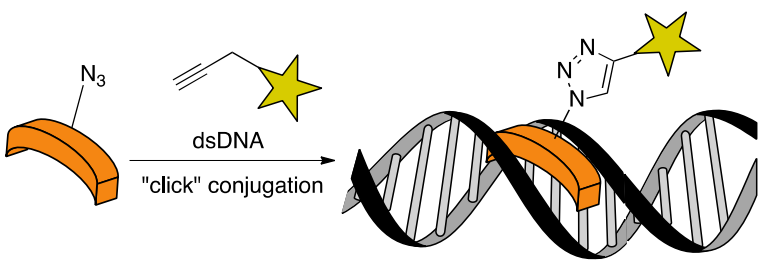

Fig 2 Strategy for sequence-specific delivery of cargoes to the vicinity of specific DNA sites.

\section{Results and Discussion \\ Synthesis of the aza-bisbenzamidines}

55 Given the commercial availability of the 4-amino benzene carboximidamide dihydrochloride (6), we designed a one-step approach to bisbenzamidines by reductive amination of suitable dialdehydes. Thus, treatment of $\mathbf{6}$ with isophthalaldehyde and $\mathrm{NaBH}_{3} \mathrm{CN}$ in $\mathrm{H}_{2} \mathrm{O} / \mathrm{MeCN}$ for $30 \mathrm{~min}$ gave the desired ${ }_{60}$ bisbenzamidine 7 in a $77 \%$ yield (Figure 3). This method could be used with other commercially available dialdehydes, therefore providing a one-step entry to a number of aza-pentamidine derivatives, which were isolated as TFA salts. Furthermore, the 1,4-benzylboronic acid $\mathbf{9}$ can be readily modified to introduce 
other groups in place of the boronic acid, adding versatility to this approach. Thus, treatment of $\mathbf{9}$ with $\mathrm{CuSO}_{4}$ and sodium azide in $\mathrm{MeOH}$ provided the azide $\mathbf{1 3}$ in good yield (86\%).

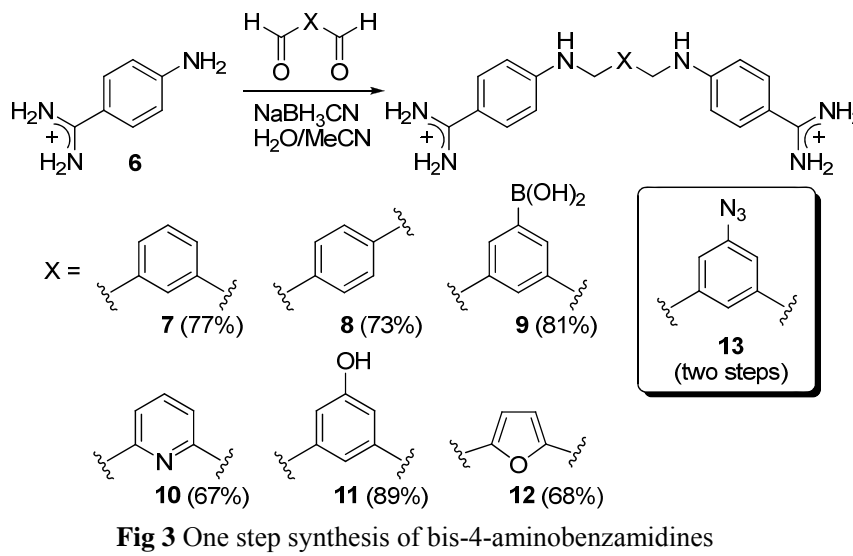

\section{DNA binding studies}

In contrast with classic bisbenzamidines, the above azabisbenzamidines exhibit a strong push/pull dipolar electronic character, and thus display environment-sensitive properties, with 10 enhanced fluorescence emission upon binding to the minor groove of their dsDNA target sites. Therefore, addition of successive aliquots of a dsDNA oligonucleotide containing a consensus AAATTT recognition site to a buffered solution of the aza-bisbenzamidine results in a progressive increase in the 15 fluorescence emission upon irradiation at $329 \mathrm{~nm}$ (Figure 4). ${ }^{9}$ These data show that the azido derivative $\mathbf{1 3}$ binds this DNA four times better than the parent meta-substituted phenyl derivative 7 , and almost one order of magnitude better than the parasubstituted phenyl derivative 8 (Table 1). ${ }^{10}$

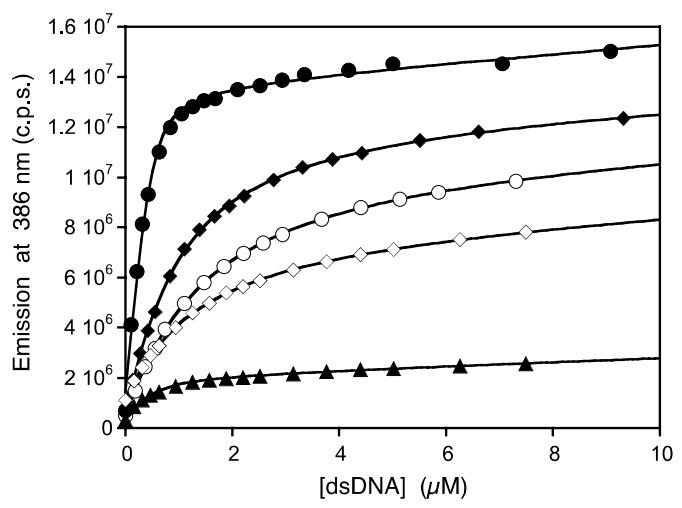

Fig 4 Titrations of selected aromatic bisbenzamidines $(0.5 \mu \mathrm{M}$ in $20 \mathrm{mM}$ Tris buffer, $100 \mathrm{mM} \mathrm{NaCl}, \mathrm{pH} 7.5)$ with a target hairpin dsDNA oligonucleotide AAATTT. $7(\bullet) ; 8(\diamond) ; 9(\circ) ; 10(\bullet)$ and $13(\bullet)$. Binding curves represent the best fit to a $1: 1$ binding mode considering the 25 contribution of the oligonucleotide to the emission. AAATTT: 5'-GGC AAATTT GAGTTTTTCTG AAATTT GCC-3' (binding site in italics).

In agreement with the selectivity exhibited by the parent azapentamidine (4), these derivatives display a clear preference for dsDNA sites containing six consecutive A/T base pairs (AAATTT) 30 over those with shorter $\mathrm{A} / \mathrm{T}$ tracts of five (AATTT), or four (AATT) pairs, ${ }^{11}$ differences which are less pronounced in the case of the azido derivative 13.
Table 1. Binding data of pentamidine derivatives 7-13

\begin{tabular}{cccc}
\hline Compound & AAATTT & AATTT & AATT \\
& $\boldsymbol{K}_{\boldsymbol{D}}$ & $\boldsymbol{K}_{\boldsymbol{D}}$ & $\boldsymbol{K}_{\boldsymbol{D}}$ \\
\hline $\mathbf{7}$ & $0.724 \pm 0.09$ & $1.2 \pm 0.1$ & $1.8 \pm 0.1$ \\
$\mathbf{8}$ & $1.3 \pm 0.2$ & $1.5 \pm 0.2$ & $1.7 \pm 0.1$ \\
$\mathbf{9}$ & $1.1 \pm 0.1$ & $1.5 \pm 0.2$ & $3.2 \pm 0.5$ \\
$\mathbf{1 0}$ & $0.229 \pm 0.063$ & $0.334 \pm 0.004$ & $1.0 \pm 0.2$ \\
$\mathbf{1 1}$ & $0.563 \pm 0.055$ & $1.0 \pm 0.1$ & $1.9 \pm 0.1$ \\
$\mathbf{1 2}$ & $0.246 \pm 0.027$ & $0.317 \pm 0.043$ & $0.782 \pm 0.064$ \\
$\mathbf{1 3}$ & $0.206 \pm 0.021$ & $0.250 \pm 0.049$ & $0.362 \pm 0.011$ \\
$\mathbf{4}$ & $0.393 \pm 0.056$ & $0.628 \pm 0.077$ & $1.1 \pm 0.1$
\end{tabular}

${ }_{35} K_{D}$ values $(\mu \mathrm{M})$ are the mean of three experiments. See the supporting information for oligo sequences and curve fitting analysis.

As expected, breaking the $\mathrm{A} / \mathrm{T}$ rich tract by introducing a single $\mathrm{A} \rightarrow \mathrm{G}$ mutation (site $A A G T T$ ) drastically reduces the stability of 40 the complexes, except for the pyridine derivative 10, which is capable of binding to this site with much better affinity $\left(K_{D} \approx 396\right.$ $\mathrm{nM})$ than the reference phenyl analog derivative $7\left(K_{D} \approx 9.1\right.$ $\mu \mathrm{M}){ }^{9}$ This result can be explained in terms of a hydrogen bond between the pyridine nitrogen and the $\mathrm{NH}_{2}$ group of the guanine, 45 and represents one of the first observations of monomeric, highaffinity binding of bisbenzamidine derivatives to sites other than pure $\mathrm{A} / \mathrm{T}$ tracts. ${ }^{12}$


Synthesis of conjugates $15 \mathrm{a}-\mathrm{c}$.

50 Fig 5 Design and click-derivatization of the aza-bisbenzamidine $\mathbf{1 3}$

Having the azide derivative $\mathbf{1 3}$ at hand, we were then challenged to demonstrate its potential as a handle for chemoselective ligations, and therefore we assembled three different conjugates using a copper-catalyzed 1,3-dipolar cycloaddition to suitable 55 alkyne-containing fragments. Each conjugate was purposely designed to demonstrate the possibilities of this DNA binder as mediator in different photophysical, physicochemical or biochemical processes. Specifically, we coupled intermediate $\mathbf{1 3}$ to a coumarine fluorophore (14a), an alkynyl-octaarginine 60 fragment $(\mathbf{1 4 b})$, and a peptide derivative (14c) that features 28 amino acids of the leucine rich region of the bZIP transcription factor cFos (Figure 5). The coupling reaction was carried out 
following standard procedures, by stirring the azide and the alkyne, in the presence of $\mathrm{CuSO}_{4}$ and sodium ascorbate. ${ }^{9,13}$
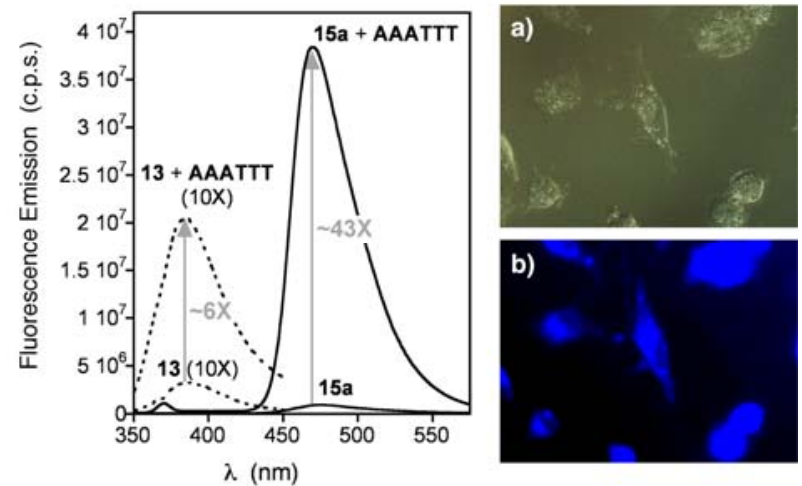

Fig 6 Left: Fluorescence emission spectra of 13 (dashed lines) and 15a 5 (solid lines) before, and after addition of a saturating concentration of the target hairpin oligonucleotide AAATTT. Spectra of $\mathbf{1 3}$ are scaled by a factor of 10 for clarity purposes. Bottom right: Incubation of 15a $(5 \mu \mathrm{M})$ with Vero cells showing effective internalization after $30 \mathrm{~min}$. a) bright field image; b) fluorescence emission of the same cells.

10 The design of the bisbenzamidine-coumarine conjugate (15a) follows our previous observations on the generation of luminescence processes through a dsDNA-dependent intramolecular energy transfer between the benzamidine and other fluorophores. ${ }^{5,14}$ Gratifyingly, while the azido15 bisbenzamidine $\mathbf{1 3}$ shows a weak fluorescence emission at 386 $\mathrm{nm}$, and only a relatively small fluorescence increase in presence of an oligonucleotide containing the A/T-rich target sequence (AAATTT), conjugate 15a exhibits a remarkably large emission increase at a longer wavelength $(\approx 470 \mathrm{~nm})$ in the presence of this ${ }_{20}$ dsDNA (Figure 6).

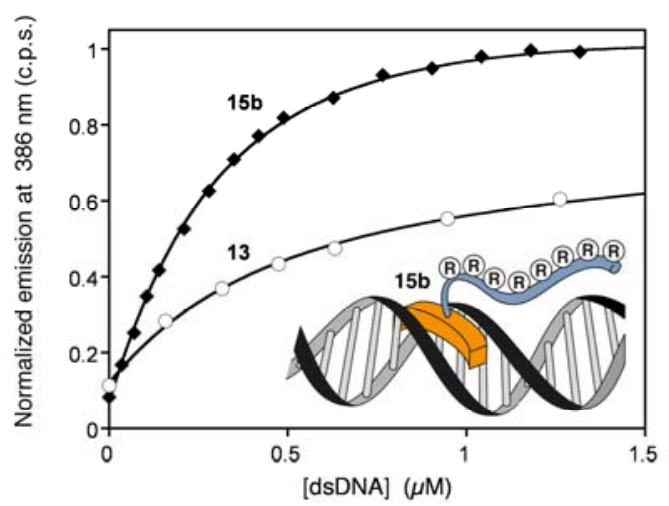

Fig 7 Fluorescence titrations of azide intermediate 13 and $\mathbf{1 5 b}(0.5 \mu \mathrm{M})$ in Tris buffer, $100 \mathrm{mM} \mathrm{NaCl}, \mathrm{pH} 7.5$ with a ds oligonucleotide featuring the AAATTT target site (see the supporting information). Proposed

25 binding of $\mathbf{1 5 b}$ to the dsDNA, showing the $(\operatorname{Arg})_{8}$ in the vicinity of the phosphates of the DNA backbone. Intensity is normalized to the saturation emission intensity of $\mathbf{1 5 b}$

In the case of the conjugate $\mathbf{1 5 b}$, we reasoned that the presence of the octaarginine tail would significantly improve the dsDNA 30 affinity of the system over an isolated bisbenzamidine. Indeed, fluorescence titrations revealed a $K_{D} \approx 35 \mathrm{nM}$ for its interaction with oligonucleotides containing a consensus A/T-rich binding site. These experiments, supported by EMSA assays (see the supporting information), also confirmed that the conjugate retains
35 the specificity of the minor groove binder (Figure 7). ${ }^{15,16}$ Remarkably, the click conjugation between the azide $\mathbf{1 3}$ and the alkyne 14b can also be carried out in the presence of the DNA. To our knowledge, this represents the first demonstration of an in situ click modification of a sequence-specific DNA binding 40 agent, and sets the basis for future DNA-guided "gain of function" applications.


Fig 8 Top: Schematic representation of the proposed interaction between hybrid 15c and cJun in a composite DNA site featuring the consensus

45 recognition sites. Bottom: EMSA of hybrid 15c demonstrating binding to the composite site. Lanes 1-4, $50 \mathrm{nM}$ ds-oligonucleotide ATF with increasing equimolar concentrations of cFos and cJun: $0,250,500$ and $1000 \mathrm{nM}$; lane 5: $50 \mathrm{nM}$ ATFhs•A/T oligonucleotide; lanes 6-8, $50 \mathrm{nM}$ ATFhs $\bullet \mathbf{A} / \mathbf{T}$ oligonucleotide with $300 \mathrm{nM}$ of cJun and increasing 50 concentrations of 15c: $0,500,1000 \mathrm{nM}$; lanes $9-11,50 \mathrm{nM} \mathrm{A} / \mathbf{T}$ oligonucleotide with increasing concentrations of cJun and 15c, 250, 500, $1000 \mathrm{nM}$. Gel staining with SYBR-Gold. Oligo sequences (only one strand is shown): ATF: 5'-GACG ATGAcgTCAT GTTCG-3'; A/T: 5'-GAC AAATTT GAGAGTACGCT-3'; ATFhs•A/T: 5'-GACG ${ }_{55}$ ATGAcgAAATTT GTTCG-3'.

Finally, conjugate 15c was designed as an artificial mimetic of the DNA binding domain of cFos, a transcription factor which specifically binds the AP1 (5'-ATGAcTCAT-3') or ATF/CREB (5'-ATGAcgTCAT-3') DNA sites as a leucine zipper-mediated 60 heterodimer with cJun. ${ }^{17}$ This heterodimer plays a fundamental role in regulating key genes involved in cell proliferation processes. ${ }^{18}$ As all members of the bZIP transcription factor family, monomeric cFos or cJun are unable to form stable DNA complexes, and high affinity DNA recognition requires their ${ }_{65}$ cooperative dimerization through a coiled-coil motive. ${ }^{19}$ Conjugate 15c, designed on the basis of the crystal structures of both the $\mathrm{cFos} / \mathrm{cJun}$ heterodimer and pentamidine bound to their respective DNA consensus sites, ${ }^{17,3 \mathrm{~b}}$ aims at stabilizing the cJun/DNA interaction but, in contrast with natural cFos, 15c 70 delivers the cFos leucine-rich region from the DNA minor groove. Furthermore, the composite dimer cJun/15c targets a DNA sequence different from that of natural AP1 or ATF/CREB sites (Figure 8).

Control EMSA experiments with cJun, $\mathrm{cFos}$ and a double 
stranded-oligonucleotide containing the target ATF recognition site revealed the formation of the expected DNA complex of the cFos/cJun heterodimer (Figure 8, lanes 2-4, band a), together with smaller amounts of DNA complexes of homodimeric cJun/cJun 5 and $\mathrm{cFos} / \mathrm{cFos}$ complexes (Figure 8, lanes 2-4, bands b and $\mathrm{c}$, respectively), in agreement with their relative stabilities. ${ }^{17}$ Gratifyingly, while cJun cannot form stable complexes with the oligonucleotide ATFhs $\bullet \mathbf{A} / \mathbf{T}$, featuring the consensus ATF half site and the bisbenzamidine preferred binding site (ATGA $\mathrm{cg}$ 10 AAATTT), incubation of that mixture with increasing concentrations of conjugate 15c generates a new slow-migrating band (Lanes 6-8, band d), consistent with the formation of the expected ternary complex between the DNA, cJun and our artificial construct 15c. Moreover, the interaction is sequenceis selective, as oligonucleotides lacking the peptide target site (like $\mathbf{A} / \mathbf{T}$ ), or with a misplaced target sites (see the supporting information), fail to provide retarded bands.

These results agree with the proposed interaction model, and confirm the potential of using minor groove binders as DNA 20 anchors to deliver peptides with designed supramolecular properties into specific DNA sites.

\section{Conclusions}

In summary, we have developed a one step, versatile protocol for the synthesis of a series of new fluorogenic, sequence-selective

${ }_{25}$ DNA binding molecules. The strategy allows a simple access to a bisbenzamidine derivative (13), which delivers an azide moiety to the external face of the DNA minor groove. The azide represents an excellent handle for bioorthogonal conjugation to different alkyne partners, providing for a number of different DNA 30 associated properties. Current efforts are focused on the extension of this supramolecular strategy to other functional systems, for non-covalent decoration of specific dsDNA sites, and for obtaining bioactive conjugates.

\section{Acknowledgements}

${ }_{35}$ We thank the support given by the Spanish grants SAF201020822-C02, CTQ2009-14431/BQU, Consolider Ingenio 2010 CSD2007-00006, and the Xunta de Galicia INCITE09 209 084PR, GRC2010/12, PGIDIT08CSA-047209PR. M. I. S. and O. $\mathrm{V}$. thank the Spanish MEC for their PhD fellowships.

\section{${ }_{40}$ Notes and references}

† Departamento de Química Orgánica y Centro Singular de Investigación en Química Biológica y Materiales Moleculares, Unidad Asociada al CSIC. Universidade de Santiago de Compostela. 15782 Santiago de Compostela (Spain). Tel: (+34) 8818144 05. E-mail:

45 joseluis.mascarenas@usc.es; or eugenio.vazquez@usc.es

"Departamento de Bioquímica y Biología Molecular y Centro Singular de Investigación en Química Biológica y Materiales Moleculares.

Universidade de Santiago de Compostela. 15782 Santiago de Compostela (Spain).

$50 \uparrow$ Electronic Supplementary Information (ESI) available: Synthesis and characterization of the bis-benzamidines and required precursors, as well as the peptide derivatives. NMR, UV, and Fluorescence spectra, fluorescent DNA titrations, gel shift assays, detailed procedure for cell uptake experiments, and molecular modeling. See 55 DOI: $10.1039 / \mathrm{b} 000000 \mathrm{x}$
1 a) J. T. Koh, J. Zheng, ACS Chem Biol. 2007, 2, 599; b) B. S. Reddy, S. M. Sondhi, J. W. Lown, Pharmacol. Ther. 1999, 84, 1; c) A. K. Mapp, Org. Biomol. Chem. 2003, 1, 2217; d) S. M. Nelson, L. R. Ferguson, W. A. Denny, Mutat. Res. 2007, 623, 24.

2 a) P. B. Dervan, R. W. Bürli, Curr. Opin. Chem. Biol. 1999, 3, 688; b) P. B. Dervan, Bioorg. Med. Chem. 2001, 9, 2215; c) A. L. Kahane, T. C. Bruice, Bioorg. Med. Chem. Lett. 2006, 16, 6255.

3 a) Z: Diwu, C. Zhang, D. H. Klaubert, R. P. Haugland, J. Photochem. Photobiol. A 2000, 131, 95; b) K. J. Edwards, T. C. Jen-kins, S. Neidle, Biochemistry 1992, 31, 7104 (Protein Data Bank ID: 1D64); c) C. M. Nunn, T. C. Jenkins, S. Neidle, Biochemistry 1993, 32, 13838.

4 a) W: D. Wilson, F. A. Tanious, A. Mathis, D. Tevis, J. E. Hall, W D. Boykin, Biochimie 2008, 90, 999; b) J. K. Thuita, S. M. Karanja, T. Wenzler, R. E. Mdachi, J. M. Ngotho, J. M. Kagira, R. Tidwell, R. Brun, Acta Trop. 2008, 108, 6; c) S. Neidle, L. R. Kelland, J. O. Trent, I. J. Simpson, D. W. Boykin, A. Kumar, W. D. Wilson, Bioorg. Med. Chem. 1997, 7, 1403; d) G. Susbielle, R. Blattes, V. Brevet, C. Monod, E. Kas, Curr. Med. Chem. Anticancer Agents 2005, 5, 409.

5 a) O. Vázquez, M. I. Sánchez, J. Martínez-Costas, J. L. Mascare-ñas, M. E. Vázquez, Chem. Commun. 2010, 46, 5518; b) O. Vázquez, M. I. Sánchez, J. Martínez-Costas, M. E. Vázquez, J. L. Mascareñas, Org. Lett. 2010, 12, 216

6 Other bisbenzamidine derivatives have been developed over the years: a) A. Mayence, J. J. V. Eynde, F. M. Krogstad, D. J. Krogstad, M. T. Cushion, T. L. Huang, J. Med. Chem. 2004, 47, 2700; b) F. Rodríguez, I. Rozas, M. Kaiser, R. Brun, B. Nguyen, W. D. Wilson, R. N. García, C. Dardonville, J. Med. Chem. 2008, 51, 909.

7 a) R. L. Staford, P. B. Dervan, J. Am. Chem. Soc. 2007, 129, 14026; b) H.-D. Arndt, K. E. Hauschild, D. P. Sullivan, K. Lake, P. B. Dervan, A. Z. Ansari, J. Am. Chem. Soc. 2003, 125, 13322; c) V. C. Rucker, S. Foister, C. Melander, P. B. Dervan, J. Am. Chem. Soc. 2003, 125, 1195; d) M. E. Vázquez, A. M. Caamaño, J. MartínezCostas, L. Castedo, J. L. Mascareñas, Angew. Chem. Int. Ed. 2001, 40, 4723; e) M. E. Vázquez, A. M. Caamaño, J. L. Mascareñas, Chem. Soc. Rev. 2003, 32, 338; f) E. Pazos, J. Mosquera, M. E. Vázquez, J. L. Mascareñas, ChemBioChem. 2011 12, 1958.

8 For postsynthetic modification of DNA using click chemistry, see: a) P. M. E. Gramlich, S. Warncke, J. Gierlich, T. Carell, Angew. Chem. Int. Ed. 2008, 47, 3442; b) P. M. E. Gramlich, C. T. Wirges, A. Manetto, T. Carell, Angew. Chem. Int. Ed. 2008, 47, 8350, and references therein.

9 See the ESI for further information.

10 The lower fluorescence intensity observed in the case of 13 can be related to the quenching effect of the azide over the fluorescence of the adjacent aza-benzamidine, as previously observed for other fluorophores: a) K. Sivakumar, F. Xie, B. M. Cash, S. Long, A. N. Barnhill, Q. Wang, Org. Lett. 2004, 6, 4603; b) F. Xie, K. Sivakumar, Q. Zeng, M. A. Bruckman, B. Hodges, Q. Wang, Tetrahedron 2008, 64, 2906.

11 Propamidines, which feature a shorter linker between the benzamidine units, tend to bind sequences of four or five $\mathrm{A} / \mathrm{T}$ base pairs, while larger $\mathrm{A} / \mathrm{T}$ tracts might accommodate more than one propamidine.

12 a) M. Rahimian, A. Kumar, M. Say, S. A. Bakunov, D. W. Boykin, R. R. Tidwell, W. D. Wilson, Biochemistry 2009, 48, 1573; b) P. Peixoto, Y. Liu, S. Depauw, M. P. Hildebrand, D. W. Boykin, C. Bailly, W. D. Wilson, M.-H. David-Cordonnier, Nucleic Acids Res. 2008, 36, 3341; c) M. Munde, M. A. Ismail, R. Arafa, P. Peixoto, C J. Collar, Y. Liu, L. Hu, M.-H. David-Cordonnier, A. Lansiaux, C. Bailly, D. W. Boykin, W. D. Wilson, J. Am. Chem. Soc. 2007, 129, 13732.

13 a) V. V. Rostovtsev, L. G. Green, V. V. Fokin, K. B.Sharpless, Angew. Chem. Int. Ed. 2002, 41, 2596; b) P. Wu, V. Fokin, Aldrichim. Acta 2007, 40, 7.

14 The coumarin acceptor was chosen considering the good spec-tral overlap between its absorption $(\lambda \mathrm{ex} \approx 409 \mathrm{~nm})$ and the emission band 
of $13(\lambda \mathrm{em} \approx 386 \mathrm{~nm})$. a) H. Zhang, T. Yu, Y. Zhao, D. Fan, L. Chem. Y. Qiu, L. Qian, K. Zhang, C. Yang, Spectrochim. Acta, A 2008, 69, 1136; b) E: Pazos, O. Vázquez, J. L. Mascareñas, M. E. Vázquez, Chem. Soc. Rev. 2009, 38, 3348.

15 O. Vázquez, J. B. Blanco-Canosa, M. E. Vázquez, J. MartínezCostas, L. Castedo, J. L. Mascareñas, ChemBioChem 2008, 9, 2822.

16 a) K. A. Browne, H.-X. He, T. C. Bruice, J. Am. Chem. Soc. 1993, 115, 7072; b) A. L. Satz, T. C. Bruice, Acc. Chem. Res. 2002, 35, 86.
17 J. N. M. Glover, S. C. Harrison, Nature 1995, 373, 257 (Protein Data Bank ID: 1FOS).

18 a) Y. Chinenov, T. K. Kerppola, Oncogene 2001, 20, 2438; b) H. van Dam, M. Castellazzi, Oncogene 2001, 20, 2453.

19 On the role of the leucine zipper, sequences of Fos and Jun proteins, and sensing applications: a) L. Chen, J. N. M. Glover, P. G. Hogan, A. Rao, S. C. Harrison, Nature 1998, 392, 42; b) E. Pazos, A. Jiménez-Balsa, J. L. Mascareñas, M. E. Vázquez, Chem. Sci. 2011, 2, 1984 